\title{
Correspondence:
}

\section{Importance of stroma in breast cancer}

Breast cancer is by far the most common diagnosed form of cancer and the leading cause of cancer death in women today. In recent years impressive advances have been made in molecular biology. However, breast cancer still remains as one of the most common malignancies because of our paucity of knowledge of what causes the disease and measures required to stop the basic pathogenic process.

Besides the various genetic and environmental factors thought to cause breast cancer, the breast tissue stroma can provide tumour suppressing and tumour promoting environments that serve to regulate neoplastic growth of the epithelium. The importance of the stroma was noticed as early as 1921 where it was clearly mentioned as "anaemia disease" in which all pigment factors were present in the body in large excess but with a scarcity of stroma-building material or an abnormality of stroma-building cells. ${ }^{2}$ The role of stroma as a mediator of normal mammary development, as well as a critical regulator of malignant conversion and progression in breast cancer is known. It is well established that stroma associated with normal mammary gland development is strikingly different from that associated with breast cancer. ${ }^{1}$ Recognition of the important role of the stroma during the progression of breast cancer leads to the possible identification of new targets for treatment of the initial breast cancer lesion as well as prevention of recurrence. Clinically useful biomarkers for early detection of breast cancer could lead to a significant reduction in mortality.

Received: 31 July, 2013.
Researchers have known for many years that a tumour is more than a homogeneous mass of cancer cells. In tumours several other cells, including fibroblasts, inflammatory immune cells, such as, macrophages, smooth muscle and endothelial cells of the blood vessels are all embedded in an extra-cellular matrix (ECM) that fibroblasts produce. Cancer researchers paid little attention to this tumour microenvironment, or "stroma", until the mid to late 1990s. The ECM does not seem to be just pretty fibrils. The ECM proteins are important in providing structural support. ${ }^{3}$ ECM proteins typically include multiple, independently folded domains whose sequences and arrangement are highly conserved. Some of these domains bind adhesion receptors such as integrins that mediate cell-matrix adhesion and also transduce signals into cells. However, ECM proteins regulate their distribution, activation, and presentation to cells. ECM proteins can integrate complex, multivalent signals to cells in a spatially patterned and regulated fashion. These properties need to be incorporated into considerations of the functions of the ECM. ${ }^{4}$

The ECM is well known for its ability to provide structural support for organs and tissues, for cell layers in the form of basement membranes, and for individual cells as substrates for migration. The role of the ECM in cell adhesion and signalling to cells through adhesion receptors such as integrins has received much attention. ${ }^{5,6}$

The stroma does not only help cancer cells to grow; it can also help them to metastasize. A 
group of enzymes called the matrix metalloproteinases (MMPs) came in for attention as researchers found that some of them could help cancer cells spread by breaking down the ECM and other barriers that would otherwise hold the cells in place. This early work culminated in clinical trials conducted primarily in the 1990 s to test whether MMP inhibitors could extend life in human patients. But the trials were spectacular failures. ${ }^{7}$ Now, however, MMPs have been identified as mediators of the communication between tumours and their microenvironment. ${ }^{8} \mathrm{MMPs}$ are largely produced by various stromal cells rather than by the tumour cells themselves. The enzymes can appear early in tumour development and may contribute to tumour growth and spread in several ways. Recently it was shown that by targetting stroma cells, wellestablished tumours could be eradicated in mice even though the tumour cells expressed little antigen. The tumours were first treated with local radiation or chemotherapy. Although this did not eliminate the tumours, it apparently killed enough cells so that their antigens were picked up by the stroma. Subsequent injection of killer T-cells destroyed both the stroma and the tumour cells. ${ }^{9}$

Another recent observation suggested that the tumour microenvironment to play an important role in invasion and metastasis in fibroblast cultured with melanoma cell lines. ${ }^{10}$ Data also suggest that stroma surrounding cancer cells plays an important role in the development and subsequent behaviour of the tumour. Studies using a wide range of techniques, including stromal cell isolation, modification of stromalspecific gene expression, and recreation of specific microenvironment in tissue samples, have demonstrated that stroma can promote cancer and that the expression patterns within the stroma can influence clinical outcome. ${ }^{9,10}$

Understanding distant metastases progression and tumour therapeutics suggest that both the stroma and epithelium may have more clinical efficacy for prevention of local recurrence and metastases. Observing the interactions of the mammary epithelial cells and its associated stroma during normal development and tumourigenesis may provide critical insight to additional chemotherapeutic targets for future therapeutics. The heightened awareness of the stroma as an active participant in carcinogenesis has led to ideas for intervening in breast cancer progression by manipulating the stroma. Compared with the multiple routes taken by cells to become cancers, the response of tissues to cancer is relatively predictable. Controlling the early stages of invasive cancer growth may therefore be more readily achieved indirectly via the stroma.

Viewing the opportunities for inhibiting cancer progression as a dynamic process, in which tumor control may be mediated by more than cell killing as a consequence of single and multiple anticancer agent exposures, may uncover additional tissue processes that are susceptible to intervention. To exploit these possibilities, we need to better understand the dynamic interactions between the epithelium and the stroma, and between cancer and the stroma, which in turn will help define the windows of opportunity in the stroma for cancer suppression and repression. This will also improve our understanding about mechanism about the stroma and suggest a novel drug targets for breast cancer.

\section{REFERENCES}

1. Bissell MJ, Radisky D. Putting tumours in context. Nat Rev Cancer 2001;1:46-54.

2. The Nobel Prize in Physiology or Medicine 1934. Available at URL: http://nobelprize.org/ nobel_prizes/medicine/laureates/1934/. Accessed on 15 October, 2013.

3. Wiseman BS, Werb Z. Stromal effects on mammary gland development and breast cancer. Science 2002;296:1046-9. 
4. Berrier AL, Yamada KM. Cell-matrix adhesion. J Cell Physiol 2007;213:565-73.

5. Legate KR, Wickström SA, Fässler R. Genetic and cell biological analysis of integrin outside-in signaling. Genes Dev 2009;23:397-418.

6. Discher DE, Mooney DJ, Zandstra PW. Growth factors, matrices, and forces combine and control stem cells. Science 2009;324:1673-7.

7. Jean Marx, All in the stroma: cancer's Cosa Nostra, Science 2008;320:38-41.

8. Ronnov-Jessen L, Petersen OW, Koteliansky VE, Bissell MJ. The origin of the myofibroblasts in breast cancer. Recapitulation of tumor environment in culture unravels diversity and implicates converted fibroblasts and recruited smooth muscle cells. J Clin Invest 1995;95:859-73.

9. West RB, Van de Rijn M. Experimental approaches to the study of cancer-stroma interactions: recent findings suggest a pivotal role for stroma in carcinogenesis, Lab Invest 2007;87:967-70.

10. Li L, Dragulev B, Zigrino P, Mauch C, Fox JW. The invasive potential of human melanoma cell lines correlates with their ability to alter fibroblast gene expression in vitro and the stromal microenvironment in vivo. Int $\mathrm{J}$ Cancer 2009;125:1796-804.

\section{A. Lavakumar Reddy, ${ }^{1}$} Kishore Polireddy. ${ }^{2}$

Departments of ${ }^{1}$ Molecular and Integrative Physiology,

${ }^{2}$ Pharmacology, Toxicology, and Therapeutics

University of Kansas Medical Center,

Kansas City, Kansas, USA 